\title{
AVALIAÇÃO DE CULTIVARES DE SOJA [Glycine max (L.) MERRILL] PARA O SUL DE MINAS GERAIS
}

\author{
Evaluation of soybean cultivars [Glycine max (L.) Merrill] for the \\ Southern of Minas Gerais State
}

\author{
Pedro Milanez de Rezende', Eudes de Arruda Carvalho
}

\begin{abstract}
RESUMO
Visando gerar informações que auxiliem técnicos e produtores na escolha da cultivar a ser utilizada em Lavras e região em plantio de verão, foi desenvolvido o presente trabalho no campo experimental da Universidade Federal de Lavras, no ano agrícola 2002/03, utilizando-se delineamento de blocos casualizados com três repetições e 45 tratamentos, constituídos pelas cultivares: Doko RC, Pioneira, Virtuosa, Conquista, FT-104, Monarca, Monsoy 108, Monsoy 109, FT-Abyara, Performa, Liderança, Monsoy 8400, Confiança, Splendor, UFV-16, Garantia, Renascença, IAC-19, FT-2000, IAC-21, CAC-1, Monsoy 8411, Suprema, Segurança, Aventis 7002, Paiaguás, Carrera, Santa Rosa, BRS - Celeste, DM 339, BRS Carla, BRS Milena, Vencedora, BR-9 Savana, Aventis 2056-7, STTE 02, Aventis 1043, Monsoy 8866, Monsoy 8329, Monsoy 9010, Embrapa 48, Emgopa 313, Emgopa 314, Tucano e BRS 136. As cultivares apresentaram produtividades satisfatórias, com destaque para Vencedora (4.395 kg.ha-1), Paiaguás (3.897 kg.ha-1), Aventis 2056-7 (3.780 kg.ha-1), Monarca $\left(3.646 \mathrm{~kg}^{-1} \mathrm{ha}^{-1}\right)$ e FT $2000\left(3.498 \mathrm{~kg} \cdot \mathrm{ha}^{-1}\right)$. Altura da planta e inserção do primeiro legume variaram de 0.68 a $1,53 \mathrm{~m}$ e de 0.08 a $0.29 \mathrm{~m}$, respectivamente. $\mathrm{O}$ índice de acamamento foi satisfatório para todas cultivares testadas. A qualidade da semente foi avaliada através do critério de notas variando de 1 a 2.3 numa escala de 1 a 5 .
\end{abstract}

Termos para indexação: Avaliação, soja.

\section{ABSTRACT}

In order to generate information to help scientists technicians and producers in the determination of cultivars to be grown in summer in Lavras region, the present work was carried out at the experimental area of Federal University of Lavras, Lavras, MG, Brazil on a Red Latosol (LVdf), in 2002/2003 crop season. The experimental design was composed in randomized blocks with three replications. The 45 cultivars (treatments) used in the experiment were: Doko, Pioneira, Virtuosa, Conquista, FT-104, Monarca, Monsoy 108, Monsoy 109, FT-Abyara, Performa, Liderança, Monsoy 8400, Confiança, Splendor, UFV-16, Garantia, Renascença, IAC-19, FT-2000, IAC-21, CAC-1, Monsoy 8411, Suprema, Segurança, Aventis 7002, Paiaguás, Carrera, Santa Rosa, BRS-Celeste, DM 339, BRS Carla, BRS Milena, BRS MG 68 (Vencedora), BR-9 Savana, Aventis 2056-7, STTE 02, Aventis 1043, Monsoy 8866, Monsoy 8329, Monsoy 9010, Embrapa 48, Emgopa 313, Emgopa 314, Tucano, BRS 136. All cultivars showed good grain yields, specially BRS MG 68 (4395 kg.ha-1), Paiaguás (3897 kg.ha-1), Aventis 2056-7 (3780 kg.ha- $\left.{ }^{-1}\right)$, Monarca (3646 kg.ha-1) and FT 2000 (3498 kg.ha-1). Soybean plant and first pod height, taken by harvest time, was in the range of $0,68 \mathrm{~m}$ to $1,53 \mathrm{~m}$ and of $0,08 \mathrm{~m}$ to $0,29 \mathrm{~m}$, respectively. Lodging indexes were also satisfactory for all cultivars tested. Seed quality of the cultivars were evaluated and ranged from 1,0 to 2,3 in a $1-5$ scale.

Index terms: Selection, soybean.

(Recebido em 19 de julho de 2005 e aprovado em 14 de agosto de 2006)

\section{INTRODUÇÃO}

A soja foi responsável pela formação de uma complexa estrutura de produção, armazenamento, processamento e de comercialização em todos os países onde é cultivada em larga escala. A grande demanda no mercado internacional proporcionou rápida expansão dessa cultura no Brasil, que ocorreu pela tomada de áreas cultivadas com outras culturas e, principalmente, da conquista de novas fronteiras agrícolas.
Dentre os fatores que atuam diretamente na cultura o fotoperíodo pode ser limitante com respeito à introdução de novas seleções em diferentes latitudes e, conforme Dutra (1986), muitas cultivares possuem uma faixa de época de plantio muito restrita, em virtude da resposta ao fotoperíodo. Como esse fator varia também com a latitude, a introdução de cultivares em determinada região deve levar em consideração o grau de sensibilidade dessas cultivares. Tal sensibilidade é característica variável entre

\footnotetext{
'Engenheiro Agrônomo, Doutor, Professor Titular - Departamento de Agricultura/DAG - Universidade Federal de Lavras/UFLA - Cx. P. 3037 - $37200-000$ Lavras, MG - pmrezend@ufla.br - Bolsista do CNPq 
cultivares, ou seja, cada uma possui seu fotoperíodo crítico, acima do qual o florescimento é atrasado. Assim cultivares das regiões de grande latitude florescem muito cedo quando cultivadas nas regiões de dia curto (VERNETTI, 1983).

Outros fatores como temperatura, umidade, fertilidade do solo, época de semeadura e densidade de plantas também afetam a altura de planta, o grau de acamamento e a produtividade (SEDIYAMA, 1989). Segundo Yuyama (1991), para uma planta manifestar o seu máximo potencial genético, caracterizado pelo seu melhor crescimento e desenvolvimento, diversos fatores ambientais podem influenciar diretamente no processo, como fotoperíodo, temperatura, radiação solar, nutrientes e vento.

A previsão de comportamento de cultivares de soja em um determinado local é dificultada, visto que em latitudes semelhantes ocorrem disponibilidade térmicas diferentes, tornando-se necessário a realização de ensaios de campo para se conhecer a fenologia das diferentes cultivares (VERNETTI, 1983). Sendo assim, diversos trabalhos vêm sendo realizados com o intuito de avaliar a adaptabilidade de genótipos a diferentes ambientes. Schoffel (2001), utilizando as cultivares Dourados, IAC 20 e IAC 28, em experimento instalado no Estado de São Paulo, constatou que tanto em período normal de semeadura como em semeaduras atrasadas, os maiores rendimentos foram obtidos com a cultivar Dourados. Em experimento instalado na ESALQ em Piracicaba, Marchiori (1998), utilizando quinze tratamentos; três cultivares (IAC 12, IAC 17 e IAC 19), e cinco populações $(200,300,400,500$ e $600 \mathrm{mil}$ plantas.ha $^{-1}$ ) verificou que as cultivares IAC 12 e IAC 19 foram as que apresentaram as maiores produtividades, ambas com rendimento acima de $2500 \mathrm{~kg} \cdot \mathrm{ha}^{-1}$.

Dado a escassez de trabalhos na região e na expectativa de fornecer subsídios para uma escolha adequada de cultivares, com o presente trabalho objetivou-se verificar o comportamento de 45 cultivares em cultivo de verão na região de Lavras-MG.

\section{MATERIAL E MÉTODOS}

O ensaio foi instalado na Universidade Federal de Lavras, situada à latitude de $21^{\circ} 14^{\prime} \mathrm{S}$, longitude $45^{\circ} 00 \mathrm{~W}$ e altitude de 918 m, conduzido no ano agrícola 2002/03 em solo classificado como Latossolo Vermelho Distroférrico típico (LVdf), cujas características químicas e físicas são descritas no Quadro1.

O preparo do solo, realizado em novembro, constitui-se de uma aração seguida de duas gradagens e a abertura de sulcos de semeadura foi realizada utilizandose tração mecânica. $\mathrm{Na}$ adubação de plantio foram aplicados $120 \mathrm{~kg} \cdot \mathrm{ha}^{-1}$ de $\mathrm{P}_{2} \mathrm{O}_{5}$ e $60 \mathrm{~kg} \cdot \mathrm{ha}^{-1}$ de $\mathrm{K}_{2} \mathrm{O}$ distribuídos e incorporados aos sulcos. As sementes foram inoculadas com Bradyrhizobium japonicum, utilizandose inoculante turfoso na proporção de $125 \mathrm{~g}$ de inoculante por $50 \mathrm{~kg}$ de semente.

A semeadura foi realizada em 11/11/02 e os desbastes nas parcelas aos 15 dias após a emergência, deixando 16 plantas por metro. Tratos culturais foram realizados em todos os tratamentos de acordo com exigências da cultura. Os índices pluviométricos e as temperaturas médias registrados em Lavras, durante a condução do ensaio, podem ser visualizados na Figura 1.

Quadro 1 -Análise química e física do solo da área experimental, ano agrícola 2002/2003, UFLA, Lavras-MG. ${ }^{1}$

\begin{tabular}{|lcc|}
\hline Características & Resultados & Interpretação \\
\hline $\mathrm{pH}$ em água $(1: 25)$ & 5,9 & Acidez média \\
$\mathrm{Ca}^{++}\left(\mathrm{cmolc}^{+} / \mathrm{dm}^{3}\right)$ & 1,5 & Médio \\
$\mathrm{Mg}^{++}\left(\mathrm{cmolc}^{3} \mathrm{dm}^{3}\right)$ & 0,4 & Baixo \\
$\mathrm{K}^{+}$disponível $\left(\mathrm{mg} / \mathrm{dm}^{3}\right)$ & 25 & Baixo \\
$\mathrm{P}$ disponível $\left(\mathrm{mg} / \mathrm{dm}^{3}\right)$ & 10 & Baixo \\
$\mathrm{Al}$ trocável $(\mathrm{m} \mathrm{E} / 100 \mathrm{cc})$ & 0,2 & Baixo \\
$\mathrm{MO}(\%)$ & 1,8 & Média \\
Areia & 16 & \\
Limo & 20 & \\
Argila & 64 & \\
\hline
\end{tabular}

1 - Análises realizadas no Laboratório "John Weelock" do Departamento de Ciência do Solo da UFLA, Lavras - MG, e as interpretações de acordo com a CFSEMG (1999). 
As parcelas experimentais foram constituídas por quatro fileiras de $5 \mathrm{~m}$ de comprimento, espaçadas por 0,50 $\mathrm{m}$, usando-se como área útil duas fileiras centrais, com eliminação de $0,50 \mathrm{~m}$ em cada extremidade das mesmas a título de bordadura.

O delineamento experimental utilizado foi o de blocos casualizados (DBC), com 3 repetições, tendo como tratamentos as 45 cultivares: Embrapa 20 (Doko RC), Pioneira, Virtuosa, Conquista, FT-104, Monarca, Monsoy 108, Monsoy 109, FT Abyara, Performa, Liderança, Monsoy 8400, BRSMG Confiança, CS 201 (Splendor), UFV-16, BRSMG Garantia, BRSMG Renascença, IAC-19, FT-2000, BR/IAC-21, CAC-1, Monsoy 8411, Suprema, BRSMG Segurança, Aventis 7002, MT/BR 45 (Paiaguás), Carrera, Santa Rosa, BRS Celeste, DM 339, BRS Carla, BRS Milena, BRSMG 68 (Vencedora), BR 9 (Savana), Aventis 2056-7, STTE 02, Aventis 1043, Monsoy 8866, Monsoy 8329, Monsoy 9010, Embrapa 48, Emgopa 313, BR/Emgopa 314 (Garça Branca), BRSMT Tucano e BRS 136.

$\mathrm{Na}$ ocasião da colheita foram avaliadas as seguintes características na área útil: altura da planta, dada pela distância do colo da planta até a extremidade da haste principal, em m, medida em 5 plantas aleatoriamente; altura de inserção do primeiro legume dada pela distância do colo da planta até a extremidade inferior do primeiro legume, em $\mathrm{m}$, de 5 plantas tomadas aleatoriamente; índice de acamamento de acordo com escala de Bernard et al. (1965), em que atribuiu-se nota $1 \mathrm{com}$ todas as plantas eretas e 5 com todas as plantas acamadas, rendimento de grãos em $\mathrm{kg} \cdot \mathrm{ha}^{-1}$ após conversão para $13 \%$ de umidade e qualidade de sementes atribuindo notas 1 para muito boa e 5 para muito pobre.
As análises estatísticas foram realizadas com auxílio do software Sisvar, sendo aplicado o teste de Scott-Knott, ao nível de $5 \%$ de probabilidade para comparação de médias.

\section{RESULTADOS E DISCUSSÃO}

Os resultados das análises estatísticas realizadas para todas as características avaliadas são apresentados no Quadro 2. Observa-se que todas características analisadas foram influenciadas significativamente pelos tratamentos testados. Os resultados médios para todas as características avaliadas são apresentados no Quadro 3.

\section{Rendimento de Grãos}

A cultivar selecionada deverá apresentar, principalmente, elevado rendimento de grãos. Outros aspectos como altura de planta e de inserção do primeiro legume, resistência a doenças e pragas, qualidade de sementes e índice de acamamento, porém não devem ser menosprezados.

De uma maneira geral, todos os materiais testados apresentaram produtividades satisfatórias, variando de 2.081 a $4.395 \mathrm{~kg} \cdot \mathrm{ha}^{-1}$. Das 45 cultivares testadas 23 apresentaram dentro do mesmo patamar de produtividade acima de $3.049 \mathrm{~kg} \cdot \mathrm{ha}^{-1}$.

Essas cultivares selecionadas apresentaram ciclos médio (111-125 dias) a semitardio (126-145 dias), podendo ser empregadas em sistema de escalonamento de colheita. A semeadura de cultivares com diferentes ciclos de maturação pode prolongar o período de colheita e algumas vezes auxiliar na obtenção de sementes de melhor qualidade.

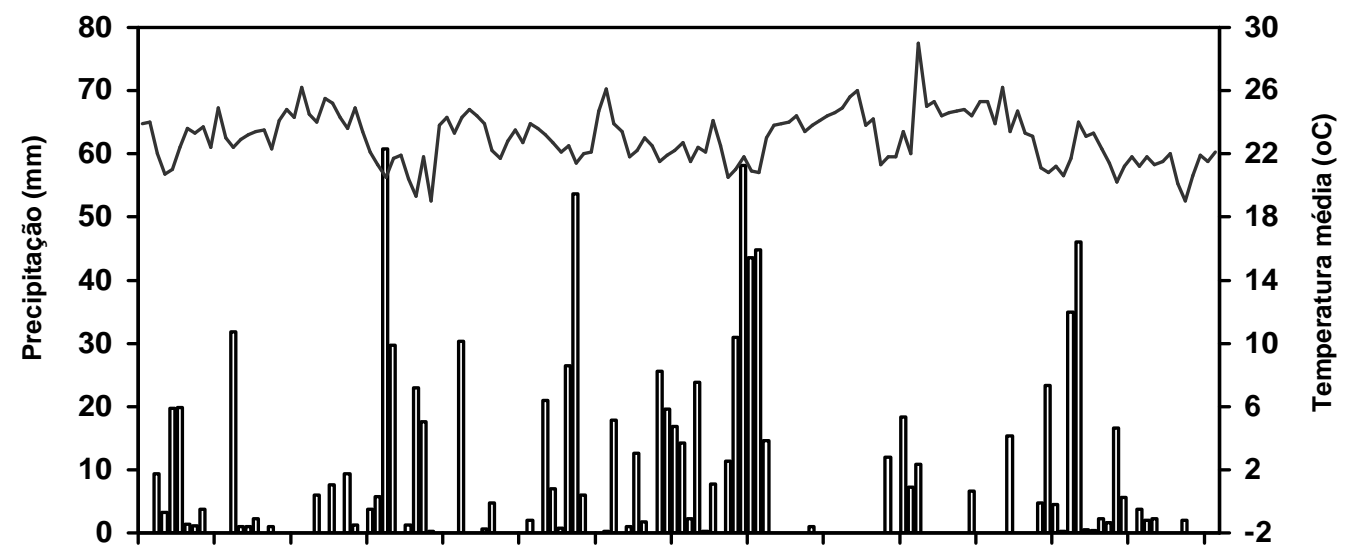

Figura 1-Variação diária da temperatura média do ar e precipitação pluvial de novembro de 2002 a março de 2003 , UFLA, Lavras (MG). Fonte: Estação Climatológica de Lavras-MG. 
Quadro 2 - Resumo da análise de variância para rendimento de grãos, altura de planta e de inserção do primeiro legume, índice de acamamento e qualidade de sementes, ensaio de seleção de cultivares, ano agrícola 2002/ 03, UFLA, Lavras - MG.*

\begin{tabular}{|c|c|c|c|c|c|c|}
\hline \multirow{3}{*}{$\begin{array}{l}\text { Causas de } \\
\text { Variação }\end{array}$} & \multirow{3}{*}{ G.L. } & \multicolumn{5}{|c|}{ Quadrados médios } \\
\hline & & \multirow{2}{*}{ Grãos } & \multicolumn{2}{|c|}{ Altura (m) } & \multirow{2}{*}{$\begin{array}{l}\text { Índice de Acam. } \\
\qquad(1 \text { a } 5)\end{array}$} & \multirow{2}{*}{$\begin{array}{l}\text { Qualidade } \\
\text { (1 a 5) }\end{array}$} \\
\hline & & & Planta & $1^{\circ}$ Legume & & \\
\hline Repetição & 2 & 618559 & 0,005 & 0,0102 & 0,0222 & 0,1407 \\
\hline Tratamentos & 44 & $636192 * *$ & $0,170 * *$ & $0,0066 * *$ & $0,7576^{* *}$ & $0,4741 *$ \\
\hline Resíduo & 88 & 297887 & 0,018 & 0,0030 & 0,1207 & 0,2771 \\
\hline C.V. & & 17,98 & 12,91 & 30,14 & 26,06 & 36,07 \\
\hline
\end{tabular}

**Significativo ao nível de $1 \%$ de probabilidade; *Significativo ao nível de $5 \%$ de probabilidade.

A semeadura em novembro, assegurada pela umidade no solo, possibilitou pleno desenvolvimento dessas cultivares. Os fatores ambientais atuantes propiciaram além de excelentes produtividades, características agronômicas satisfatórias, demonstrando boa adaptabilidade desses genótipos às condições locais.

A cultivar Liderança (ciclo médio), que não diferiu significativamente daquelas de maiores produtividades, também apresentou boas características agronômicas, podendo ser utilizada para o cultivo na região. O mesmo pode ser estendido àquelas cultivares que, de acordo com o Quadro 3, também não apresentaram diferenças significativas para rendimento de grãos, atentando-se, no entanto, às demais características de interesse.

Por outro lado, o menor rendimento de grãos foi observado na cultivar Monsoy 8866, apesar de ter apresentado boa altura de planta. Esse fato pode estar relacionado à sua má adaptação à região. Ocorreu considerável crescimento vegetativo, explicitado pela altura de planta, e indução floral tardia, poucas flores e consequientemente baixa produtividade. $\mathrm{O}$ alto índice de acamamento vem confirmar essa hipótese, uma vez que, de acordo com Sediyama et al. (1989), o acamamento pode ser indício de má adaptação ao local de cultivo. Não obstante essa não é uma cultivar recomendada para o Estado de MG. Do mesmo modo, as cultivares Pioneira e Tucano também não são recomendadas para a região onde foi conduzido o ensaio, explicitando a baixa adaptabilidade pelas características agronômicas apresentadas (Quadro
3). Relato de vários pesquisadores Dutra (1986), Peluzio (1996), Schoffel (2001) e Rocha (2002) também indicaram uma resposta diferencial das cultivares às diversas características dentro do próprio local de semeadura recomendado.

\section{Altura de Planta e de Inserção do Primeiro Legume}

A altura de planta é característica fundamental na determinação da cultivar a ser introduzida em uma região, podendo variar consideravelmente, de acordo com a época de semeadura, espaçamento de plantas entre e dentro das fileiras, suprimento de umidade, temperatura, fertilidade do solo e outras condições gerais do meio ambiente (CARTTER \& HARTWIG, 1962). Dependendo da resposta fotoperiódica da cultivar, a planta pode ter altura reduzida e formação de legumes bem próximos ao solo. Considera-se, portanto, alturas de planta compreendidas entre 60 e 120 como adequadas à mecanização da colheita.

Para Marcos Filho (1986), a variedade escolhida para cultivo numa determinada localidade deve apresentar uma altura de inserção da primeiro legume de pelo menos 10 a $12 \mathrm{~cm}$. Entretanto, segundo o autor, para a maioria das condições das lavouras de soja, a altura mais satisfatória está em torno de $15 \mathrm{~cm}$, embora colhedoras mais modernas possam efetuar boa colheita com plantas apresentando inserção de legume a $10 \mathrm{~cm}$. Os fatores ambientais ou de práticas culturais que afetam a altura de planta podem influenciar também a altura da inserção da primeira vagem (SEDIYAMA et al., 1972). 
Quadro 3 - Resultados médios do rendimentos de grãos, altura de planta e de inserção do primeiro legume, índice de acamamento e qualidade de sementes obtidos no ensaio de seleção de cultivares, ano agrícola 2002/03,UFLA, Lavras $-\mathrm{MG}^{*}$.

\begin{tabular}{|c|c|c|c|c|c|}
\hline \multirow{2}{*}{ Cultivares } & \multirow{2}{*}{ 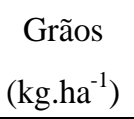 } & \multicolumn{2}{|c|}{ Altura (m) } & \multirow{2}{*}{$\begin{array}{l}\text { Índice de } \\
\text { Acamam. }\end{array}$} & \multirow{2}{*}{ Qualidade } \\
\hline & & Planta & $1^{\circ}$ Legume & & \\
\hline BRSMG 68 & $4395 \mathrm{a}$ & $1,04 \mathrm{a}$ & $0,17 \mathrm{a}$ & $1,00 \mathrm{a}$ & $1,00 \mathrm{a}$ \\
\hline Paiaguás & $3898 \mathrm{a}$ & $1,20 \mathrm{~b}$ & $0,26 \mathrm{~b}$ & $1,33 \mathrm{a}$ & $1,00 \mathrm{a}$ \\
\hline AV 2056-7 & $3780 \mathrm{a}$ & $1.40 \mathrm{c}$ & $0,15 \mathrm{a}$ & $1,33 \mathrm{a}$ & $1,67 \mathrm{~b}$ \\
\hline Monarca & $3646 \mathrm{a}$ & $1,17 \mathrm{~b}$ & $0,15 \mathrm{a}$ & $1,33 \mathrm{a}$ & $2,00 \mathrm{~b}$ \\
\hline FT - 2000 & 3498 a & $1,15 \mathrm{~b}$ & $0,19 \mathrm{~b}$ & $1,00 \mathrm{a}$ & $1,33 \mathrm{a}$ \\
\hline Santa Rosa & $3454 \mathrm{a}$ & $0,84 \mathrm{a}$ & $0,12 \mathrm{a}$ & $1,00 \mathrm{a}$ & $1,33 \mathrm{a}$ \\
\hline Emgopa 313 & $3436 \mathrm{a}$ & $1,29 \mathrm{c}$ & $0,13 \mathrm{a}$ & $2,00 \mathrm{~b}$ & $1,00 \mathrm{a}$ \\
\hline Monsoy 8329 & 3418 a & $1,15 \mathrm{~b}$ & $0,21 \mathrm{~b}$ & $1,00 \mathrm{a}$ & $1,00 \mathrm{a}$ \\
\hline IAC -21 & $3384 \mathrm{a}$ & $1,02 \mathrm{~b}$ & $0,19 \mathrm{~b}$ & $1,00 \mathrm{a}$ & $1,33 \mathrm{a}$ \\
\hline Carrera & $3382 \mathrm{a}$ & $1,04 \mathrm{~b}$ & $0,22 \mathrm{~b}$ & $1,00 \mathrm{a}$ & $1,33 \mathrm{a}$ \\
\hline BRS Carla & $3361 \mathrm{a}$ & $0,97 \mathrm{a}$ & $0,17 \mathrm{a}$ & $1,00 \mathrm{a}$ & $2,00 \mathrm{~b}$ \\
\hline IAC - 19 & 3359 a & $1,02 \mathrm{~b}$ & $0,22 \mathrm{~b}$ & $1,00 \mathrm{a}$ & $1,00 \mathrm{a}$ \\
\hline Garantia & $3317 \mathrm{a}$ & $1,06 \mathrm{~b}$ & $0,26 \mathrm{~b}$ & $1,00 \mathrm{a}$ & $1,00 \mathrm{a}$ \\
\hline AV 1043 & 3316 a & $1,53 \mathrm{c}$ & $0,20 \mathrm{~b}$ & $1,67 \mathrm{~b}$ & $1,67 \mathrm{~b}$ \\
\hline BRS 136 & 3296 a & $0,87 \mathrm{a}$ & $0,23 \mathrm{a}$ & $1,00 \mathrm{a}$ & $1,33 \mathrm{a}$ \\
\hline BRS Celeste & $3207 \mathrm{a}$ & $1,20 \mathrm{~b}$ & $0,21 \mathrm{~b}$ & $1,67 \mathrm{~b}$ & $1,33 \mathrm{a}$ \\
\hline Monsoy 109 & $3204 \mathrm{a}$ & $1,15 \mathrm{~b}$ & $0,17 \mathrm{a}$ & $1,33 \mathrm{a}$ & $2,00 \mathrm{~b}$ \\
\hline Performa & $3170 \mathrm{a}$ & $0,93 \mathrm{a}$ & $0,14 \mathrm{a}$ & $1,00 \mathrm{a}$ & $1,33 \mathrm{a}$ \\
\hline Monsoy 8400 & $3153 \mathrm{a}$ & $1,08 \mathrm{~b}$ & $0,24 \mathrm{~b}$ & $1,33 \mathrm{a}$ & $1,67 \mathrm{~b}$ \\
\hline BR-9 Savana & $3135 \mathrm{a}$ & $1,24 \mathrm{c}$ & $0,18 \mathrm{~b}$ & $1,00 \mathrm{a}$ & $1,67 \mathrm{~b}$ \\
\hline Segurança & $3074 \mathrm{a}$ & $1,12 \mathrm{~b}$ & $0,20 \mathrm{~b}$ & $1,33 \mathrm{a}$ & $1,00 \mathrm{a}$ \\
\hline $\mathrm{CAC}-1$ & 3064 a & $1,00 \mathrm{~b}$ & $0,08 \mathrm{a}$ & $1,00 \mathrm{a}$ & $1,00 \mathrm{a}$ \\
\hline Liderança & 3049 a & $1,20 \mathrm{~b}$ & $0,19 \mathrm{~b}$ & $1,00 \mathrm{a}$ & $1,67 \mathrm{~b}$ \\
\hline DM 339 & $3018 \mathrm{~b}$ & $1,23 \mathrm{~b}$ & $0,25 \mathrm{~b}$ & $1,67 \mathrm{~b}$ & $1,67 \mathrm{~b}$ \\
\hline Splendor & $2988 \mathrm{~b}$ & $1,03 \mathrm{~b}$ & $0,19 \mathrm{~b}$ & $1,00 \mathrm{a}$ & $1,33 \mathrm{a}$ \\
\hline Aventis 7002 & 2947 b & $1,14 \mathrm{~b}$ & $0,18 \mathrm{~b}$ & $1,33 \mathrm{a}$ & $1,33 \mathrm{a}$ \\
\hline Conquista & $2897 \mathrm{~b}$ & $0,97 \mathrm{a}$ & $0,20 \mathrm{~b}$ & $1,00 \mathrm{a}$ & $1,67 \mathrm{~b}$ \\
\hline
\end{tabular}


Quadro 3-Continuação...

\begin{tabular}{|c|c|c|c|c|c|}
\hline \multirow{2}{*}{ Cultivares } & \multirow{2}{*}{$\begin{array}{c}\text { Grãos } \\
\left(\mathrm{kg} \cdot \mathrm{ha}^{-1}\right)\end{array}$} & \multicolumn{2}{|c|}{ Altura (m) } & \multirow{2}{*}{$\begin{array}{l}\text { Índice de } \\
\text { Acamam. }\end{array}$} & \multirow{2}{*}{ Qualidade } \\
\hline & & Planta & $1^{\circ}$ Legume & & \\
\hline FT - Abyara & $2871 \mathrm{~b}$ & $0,77 \mathrm{a}$ & $0,13 \mathrm{a}$ & $1,00 \mathrm{a}$ & $1,33 \mathrm{a}$ \\
\hline Emgopa 314 & $2834 \mathrm{~b}$ & $1,26 \mathrm{c}$ & $0,15 \mathrm{a}$ & $2,33 \mathrm{c}$ & $1,00 \mathrm{a}$ \\
\hline Virtuosa & $2818 b$ & $0,88 \mathrm{c}$ & $0,12 \mathrm{a}$ & $1,00 \mathrm{a}$ & $2,33 \mathrm{~b}$ \\
\hline Monsoy 108 & $2756 \mathrm{~b}$ & $1,19 \mathrm{~b}$ & $0,23 \mathrm{~b}$ & $2,33 \mathrm{c}$ & $2,33 \mathrm{~b}$ \\
\hline FT - 104 & $2754 \mathrm{~b}$ & $1,17 \mathrm{~b}$ & $0,13 \mathrm{a}$ & $1,67 \mathrm{~b}$ & $1,33 \mathrm{a}$ \\
\hline STTE 02 & $2737 \mathrm{~b}$ & $0,70 \mathrm{a}$ & $0,17 \mathrm{a}$ & $1,33 \mathrm{a}$ & $1,67 \mathrm{~b}$ \\
\hline Confiança & $2726 \mathrm{~b}$ & $0,94 \mathrm{a}$ & $0,14 \mathrm{~b}$ & $1,00 \mathrm{a}$ & $1,33 \mathrm{a}$ \\
\hline BRS Milena & $2716 \mathrm{~b}$ & $1,08 \mathrm{~b}$ & $0,20 \mathrm{~b}$ & $1,00 \mathrm{a}$ & $1,67 \mathrm{~b}$ \\
\hline Monsoy 8411 & $2687 \mathrm{~b}$ & $1,10 \mathrm{~b}$ & $0,24 \mathrm{~b}$ & $1,33 \mathrm{a}$ & $1,00 \mathrm{a}$ \\
\hline Embrapa 48 & $2675 \mathrm{~b}$ & $0,69 \mathrm{a}$ & $0,09 \mathrm{a}$ & $1,00 \mathrm{a}$ & $2,00 \mathrm{~b}$ \\
\hline Renascença & $2625 \mathrm{~b}$ & $0,83 \mathrm{a}$ & $0,11 \mathrm{a}$ & $1,00 \mathrm{a}$ & $2,33 \mathrm{~b}$ \\
\hline Doko & $2598 \mathrm{~b}$ & $1,14 \mathrm{~b}$ & $0,23 \mathrm{~b}$ & $2,00 \mathrm{~b}$ & $2,00 \mathrm{a}$ \\
\hline Suprema & $2558 \mathrm{~b}$ & $1,29 \mathrm{c}$ & $0,20 \mathrm{~b}$ & $1,00 \mathrm{a}$ & $1,00 \mathrm{a}$ \\
\hline $\mathrm{UFV}-16$ & $2491 \mathrm{~b}$ & $0,91 \mathrm{a}$ & $0,20 \mathrm{~b}$ & $1,00 \mathrm{a}$ & $1,00 \mathrm{a}$ \\
\hline Monsoy 9010 & $2318 \mathrm{~b}$ & $1,13 \mathrm{~b}$ & $0,19 \mathrm{~b}$ & $3,00 \quad \mathrm{c}$ & $1,33 \mathrm{a}$ \\
\hline Tucano & $2271 \mathrm{~b}$ & $1,15 \mathrm{~b}$ & $0,14 \mathrm{a}$ & $1,33 \mathrm{a}$ & $1,67 \mathrm{~b}$ \\
\hline Pioneira & $2259 \mathrm{~b}$ & $1,35 \mathrm{c}$ & $0,29 \mathrm{~b}$ & $2,00 \mathrm{~b}$ & $1,33 \mathrm{a}$ \\
\hline Monsoy 8866 & $2081 \mathrm{~b}$ & $1,26 \mathrm{c}$ & $0,24 \mathrm{~b}$ & $2,67 \mathrm{c}$ & $1,33 \mathrm{a}$ \\
\hline
\end{tabular}

*Médias seguidas pelas mesmas letras não diferem significativamente pelo teste de Scott-Knott, ao nível de 5\% de probabilidade.

Dentre as cultivares de maior destaque, salienta-se que a Aventis 2056-7 apresentou porte muito alto, podendo neste caso ocasionar perdas na colheita mecanizada, seja pela tendência natural ao acamamento ou pelo contato com o molinete da colhedora.

\section{Acamamento}

Essa característica assume importante papel na seleção de cultivares, visto que poderá provocar perdas no processo de colheita mecanizada, juntamente com a altura de planta e de inserção do primeiro legume. No presente trabalho, verificou-se notas compreendidas entre 1 e 3, sendo os maiores valores observados para as cultivares Monsoy 9010 (3) e Monsoy 8866 (2,7), conforme indica o Quadro 3.

\section{Qualidade das sementes}

As chuvas ocorridas na pré-colheita, juntamente com a elevação da temperatura, contribuíram para a queda na qualidade de sementes, por favorecerem a infestação 
de doenças de final de ciclo. Cultivares semitardias e tardias expuseram-se mais ao ataque de percevejos durante os estádios finais de seu ciclo. Já para as demais diferenças observadas, dentro de cada grupo de maturação, acreditase que foram proporcionadas por fatores genéticos que, por interação com as condições locais, demonstraram melhor adaptabilidade de certas cultivares a estas condições.

Evidenciando-se o que foi exposto verifica-se que as cultivares BRSMG 68 (Vencedora) e Monsoy 8411 apresentaram excelente qualidade de sementes, enquanto outras de mesmo ciclo como Monsoy 109 e Renascença apresentaram sementes de pior qualidade. As médias das notas de qualidade das sementes dos genótipos estudados variaram de 1,0 a 2,3. Não foi encontrada nenhuma relação entre grupos de maturação e qualidade de sementes. Contudo, é desejável que a cultivar escolhida se desenvolva de forma que suas fases de maior exigência hídrica coincidam com os picos de índices pluviométricos e, sobretudo, alcance o ponto de colheita sob declínio de temperaturas do ar e de umidade, já que Medina (1994) verificou que cultivares precoces de soja semeadas no Estado de São Paulo durante os meses de outubro e novembro, desenvolveram seus processos de maturação e colheita sob condições climáticas desfavoráveis, caracterizadas por elevadas temperaturas e umidades relativas do ar.

\section{CONCLUSÕES}

As cultivares estudadas mostraram índice de acamamento, altura de planta e de inserção do primeiro legume favoráveis à colheita mecanizada, com exceção das cultivares CAC-1 e Embrapa 48, com alturas de inserção do primeiro legume de 8 e $9 \mathrm{~cm}$, respectivamente.

As cultivares avaliadas apresentaram excelentes produtividades variando de $3.049 \mathrm{~kg} \cdot \mathrm{ha}^{-1}$ a $4.395 \mathrm{~kg} \cdot \mathrm{ha}^{-1}$, com destaque para as cultivares BRS MG 68 (Vencedora) (4.395 kg.ha-1), Paiaguás (3.897 kg.ha' $\left.{ }^{-1}\right)$, Aventis 2056-7 (3.780 kg.ha-1), Monarca (3.646 kg.ha-1) e FT 2000 (3.498 kg.ha' $\left.{ }^{-1}\right)$.

\section{REFERÊNCIAS BIBLIOGRÁFICAS}

BERNARD, R. L.; CHAMBERLAIN, D. W.; LAWRENCE, R. D. (Eds.). Result of the cooperative uniform soybeans tests. Washington: USDA, 1965. $134 \mathrm{p}$.

CARTTER, J. L.; HARTWIG, E. E. The management of soybeans. In: NORMAN, A. G. (Ed.). The soybean. New York: Academic, 1962.
COMISSÃO DE FERTILIDADE DO SOLO DO ESTADO DE MINAS GERAIS. Recomendações para o uso de corretivos e fertilizantes em Minas Gerais: $5^{\text {a }}$ aproximação. Viçosa, 1999. 359 p.

DUTRA, J. H. Comportamento de quinze genótipos de soja [Glycine $\operatorname{Max}(\mathrm{L}$.$) Merrill], em diferentes épocas de plantio,$ em Capinópolis, Minas Gerais. 1986. 59 f. Dissertação (Mestrado) - Universidade Federal de Viçosa, Viçosa, 1986.

MARCHIORI, L. F. S. Desempenho vegetativo e produtivo de três cultivares de soja e cinco densidades populacionais nas épocas normal e safrinha. 1998. Dissertação (Mestrado em Fitotecnia) - Escola Superior de Agricultura Luiz de Queiroz, Universidade de São Paulo, Piracicaba, 1998.

MARCOS FILHO, J. Produção de sementes de soja. Campinas: Fundação Cargill, 1986. 86 p.

MEDINA, P. F. Produção de sementes de cultivares de soja em diferentes épocas e locais do estado de São Paulo. 1994. $173 \mathrm{f}$. Tese (Doutorado) - Escola superior de Agricultura Luiz de Queiroz, Universidade de São Paulo, Piracicaba, 1994.

PELUZIO, J. M. Interação cultivar x Ambiente e correlação fenotípicas, genotípicas e de ambiente entre cultivares de soja no estado de Tocantins. 1996. $80 \mathrm{f}$. Tese (Doutorado) Universidade Federal de Viçosa, Viçosa, 1996.

ROCHA, M. M. Seleção de linhagens experimentais de soja para adaptabilidade e estabilidade fenotípica. 2002. 173 f. Tese (Doutorado) - Escola Superior de Agricultura Luiz de Queiroz, Universidade de São Paulo, Piracicaba, 2002.

SCHOFFEL, E. R. Épocas de semeadura, fenologia, características fisiológicas e agronômicas de três cultivares de soja. 2001. Tese (Doutorado em Produção Vegetal) - Faculdade de Ciências Agrárias e Veterinárias, Jaboticabal, 2001.

SEDIYAMA, T.; CARDOSO, A. A.; VIEIRA, C.; ATHOW, K. L. Efeito de espaçamento entre e dentro das fileiras de plantio sobre duas variedades de soja, em Viçosa e Capinópolis. Revista Ceres, Viçosa, v. 19, n. 104, p. 89-107, 1972.

SEDIYAMA, T.; PEREIRA, M. G.; SEDIYAMA, C. S.; GOMES, J. L. L. Cultura da soja: $1^{a}$ parte. Viçosa: UFV, 1989. 
VERNETTI, F. J. Soja: planta, clima, pragas, moléstias e invasoras. Campinas: Fundação Cargill, 1983. v. 1.

YUYAMA, K. Avaliação de algumas características agronômicas e morfofisiológicas de cinco cultivares de soja (Glycine max (L.) Merrill), cultivados em solo de várzea e de terra firme da Amazônia Central. 1991. 123 f. Tese (Doutorado em Produção Vegetal) - Faculdade de Ciências Agrárias e Veterinárias, Universidade Estadual Paulista, Jaboticabal, 1991. 